\title{
Development of attenuated live vaccine candidates against swine brucellosis in a non-zoonotic $B$. suis biovar 2 background
}

\author{
Beatriz Aragón-Aranda ${ }^{1 \dagger}$, María Jesús de Miguel ${ }^{2,3 \dagger}$, Leticia Lázaro-Antón ${ }^{1}$, Miriam Salvador-Bescós ${ }^{1}$, \\ Amaia Zúñiga-Ripa', Ignacio Moriyón ${ }^{1}$, Maite Iriarte ${ }^{1}$, Pilar M. Muñoz $2,3^{*+} \oplus$ and Raquel Conde-Álvarez ${ }^{1{ }^{*+}}$
}

\begin{abstract}
Brucella is a genus of gram-negative bacteria that cause brucellosis. B. abortus and B. melitensis infect domestic ruminants while $B$. suis (biovars 1-3) infect swine, and all these bacteria but $B$. suis biovar 2 are zoonotic. Live attenuated $B$. abortus $\mathrm{S} 19$ and $B$. melitensis Rev1 are effective vaccines in domestic ruminants, though both can infect humans. However, there is no swine brucellosis vaccine. Here, we investigated the potential use as vaccines of $B$. suis biovar 2 rough (R) lipopolysaccharide (LPS) mutants totally lacking O-chain (Bs2 $\triangle w b k F$ ) or only producing internal O-chain precursors (Bs2 $\triangle w z m$ ) and mutants with a smooth (S) LPS defective in the core lateral branch (Bs2 $\triangle$ wadB and Bs $2 \triangle$ wadD). We also investigated mutants in the pyruvate phosphate dikinase (Bs $2 \Delta p p d K$ ) and phosphoenolpyruvate carboxykinase (Bs2 $\triangle p c k A$ ) genes encoding enzymes bridging phosphoenolpyruvate and the tricarboxylic acid cycle. When tested in the OIE mouse model at the recommended R or S vaccine doses $\left(10^{8}\right.$ and $10^{5} \mathrm{CFU}$, respectively), CFU/spleen of all LPS mutants were reduced with respect to the wild type and decreased faster for the $R$ than for the $S$ mutants. At those doses, protection against $B$. suis was similar for Bs $2 \Delta w b k F, B s 2 \Delta w z m, B s 2 \Delta w a d B$ and the Rev1 control $\left(10^{5} \mathrm{CFU}\right)$. As described before for B. abortus, B. suis biovar 2 carried a disabled $p c k A$ so that a double mutant Bs $2 \triangle p p d K \Delta p c k A$ had the same metabolic phenotype as $B s 2 \Delta p p d K$ and $p p d K$ mutation was enough to generate attenuation. At $10^{5} \mathrm{CFU}$, Bs2 $\triangle p p d K$ also conferred the same protection as Rev1. As compared to other B. suis vaccine candidates described before, the mutants described here simultaneously carry irreversible deletions easy to identify as vaccine markers, lack antibiotic-resistance markers and were obtained in a non-zoonotic background. Since $R$ vaccines should not elicit antibodies to the S-LPS and wZm mutants carry immunogenic O-chain precursors and did not improve Bs $2 \triangle W b k F$, the latter seems a better $R$ vaccine candidate than $B s 2 \Delta w z m$. However, taking into account that all $R$ vaccines interfere in ELISA and other widely used assays, whether Bs $2 \Delta w b k F$ is advantageous over Bs $2 \Delta w a d B$ or Bs $2 \Delta p p d K$ requires experiments in the natural host.
\end{abstract}

\footnotetext{
*Correspondence: pmmunnoz@cita-aragon.es; rconde@unav.es †Beatriz Aragón-Aranda and María Jesús de Miguel contributed equally to this work

†Pilar M. Muñoz and Raquel Conde-Álvarez are both corresponding authors and contributed equally to this work

${ }^{1}$ Instituto de Salud Tropical (ISTUN), Instituto de Investigación Sanitaria de Navarra (IdiSNA) and Dpto. de Microbiología y Parasitología, Universidad de Navarra, c/Irunlarrea 1, 31008 Pamplona, Spain ${ }^{2}$ Unidad de Producción y Sanidad Animal, Centro de Investigación y Tecnología Agroalimentaria de Aragón (CITA), Avda. Montañana 930, 50059 Zaragoza, Spain

Full list of author information is available at the end of the article
}

\section{Introduction}

Brucellosis is a worldwide extended zoonosis caused by gram-negative bacteria of the genus Brucella. This genus includes several nominal species among which $B$. melitensis preferentially infects small ruminants, $B$. abortus cattle and B. suis swine and semi-domestic and wild mammals [1]. These three species have been classically divided into biovars following phenotypic criteria [2], and out of the five biovars currently distinguished within B. suis, three infect domestic pigs. Biovars 1 and 3 
are endemic in America and Asia, affect mainly domestic (and feral) pigs and wild boars and are very pathogenic for humans. B. suis biovar 2 (henceforth bv2) causes an enzootic infection in wild boars and also in hares in continental Europe. However, in contrast to other biovars infecting swine, bv2 shows a very reduced pathogenicity (if any) for humans. Indeed, the few infections reported only affect individuals with predisposing comorbidities that have been highly exposed $[3,4]$. Although European Union countries are considered as free of porcine brucellosis, contacts between wild-life animals and domestic pigs occur in outdoor breeding systems and back yard herds, causing brucellosis outbreaks and subsequent long-term reproductive failures and economic losses [4]. In addition, $B$. suis bv2 can be introduced into intensive pig farms through infected replacements and/or semen [3].

In most cases, the complex and surreptitious dynamics of brucellosis makes the use of effective vaccines a requisite for its control and eradication in domestic animals [5]. But for a few instances where circumstances were highly favorable, the use of the B. abortus S19 (cattle live vaccine) and $B$. melitensis Rev1 (sheep and goat live vaccine) has been decisive wherever eradication has been achieved in domestic ruminants [6]. However, few studies have investigated brucellosis vaccines in swine, and none has been satisfactory. Both controlled experiments and field observations soon discarded B. abortus S19 as a useful brucellosis vaccine in pigs $[7,8]$ and, despite initial claims on full protection by the rough (R) (i.e. lacking the O-polysaccharide [O-chain] of the lipopolysaccharide [LPS]) B. abortus RB51 cattle vaccine [9], controlled experiments prove that this strain does not provide any protection to gilts [10]. Vaccination with a $B$. abortus attenuated strain ("Bang Viejo") and the simultaneous injection of a crude heat extract of $B$. suis, plus a booster with the latter 1 month later, was reported to provide protection [11]. However, further experiments under controlled conditions found that this vaccination procedure (named INTA vaccine) does not protect pigs [11]. Attempts to develop a $B$. suis specific vaccine have been made using $B$. suis biovar 1 as background. In early studies, an apparently attenuated strain (Australian King 8) induced some protection at 6 months but none at 24 months after vaccination, which together with concerns about its reversion to full virulence and likely pathogenicity to humans, discarded its use as a vaccine [7]. B. suis strain 2 (also a biovar 1 derivative obtained by serial passage in vitro) has been claimed to be very useful in swine, and also in sheep, goats and cattle, and has been used in China since 1971. There is little experience with strain 2 outside of this country and, although it has been reported that its use led to brucellosis control in several areas of China [12], this claim is neither compatible with the present situation of the disease in the country [13] nor with the assessments made in European laboratories under controlled conditions in sheep [14, 15]. To the best of our knowledge, only a subunit vaccine has been tested in pigs. Edens and Foster [16] studied materials in the water phase (erroneously assumed to be rich in LPS) of a phenol/water extract of $B$. suis and reported protection. However, the data show protection inconsistent with the doses tested, which suggests that the bacteriological procedures (only a lymph node of the cervical region was examined for the challenge strain) were not optimized.

Although not tested in pigs, there have also been attempts to develop $B$. suis vaccines taking advantage of a better understanding of Brucella virulence factors and antigens. $B$. abortus and $B$. melitensis $\mathrm{R}$ mutants defective in the $\mathrm{O}$-chain or in the $\mathrm{O}$-chain plus core sugars of the LPS are attenuated and have been extensively investigated as a way to diminish the interference of ruminant vaccines in standard serological tests [17-20]. However, only Pgm (phosphoglucomutase involved in the production of the UDP-glucose involved in bridging the LPS core to the O-chain and in the synthesis of the periplasmic glucans [21]) and WboA (O-chain glycosyltransferase) have been mutated for developing a $B$. suis vaccine, either in a $B$. suis bv1 strain [21-23] or in $B$. neotomae [24]. Whereas both types of mutants yielded some protection in mice (see "Discussion"), both are made in brucellae that are infectious for humans and do not fully explore the possibilities offered by our current understanding of the genetics and structure of Brucella LPS. RB51 carries also a disrupted $w b o A$ [19] and it has been manipulated in attempts to bolster its performance either by overexpressing proteins thought to be immunogenic or involved in virulence [25] and/or by restoring the ability to induce anti-S-LPS antibodies $[25,26]$. Yet, RB51 carries unknown genetic defects affecting LPS [19], which together with its poor performance in pigs [10] indicate that it is not an optimal background for developing a swine brucellosis vaccine. Moreover, RB51 (and hence other less attenuated $R$ vaccines) is infectious for humans, and in this context it poses two additional problems: its resistance to rifampicin (used to treat human brucellosis) and undetectability of the infection in standard brucellosis serological tests [27].

In this work, we investigated B. suis mutants that have three different types of LPS: carrying a complete LPS core but lacking the O-chain, carrying a complete LPS core and producing an internal O-chain, and mutants carrying an LPS with O-chain (i.e. a smooth [S] LPS) but defective in the core lateral branch (Figure 1A-B), three complementary strategies that produce attenuation and that respectively abrogate or not $\mathrm{O}$-chain antibody 
A

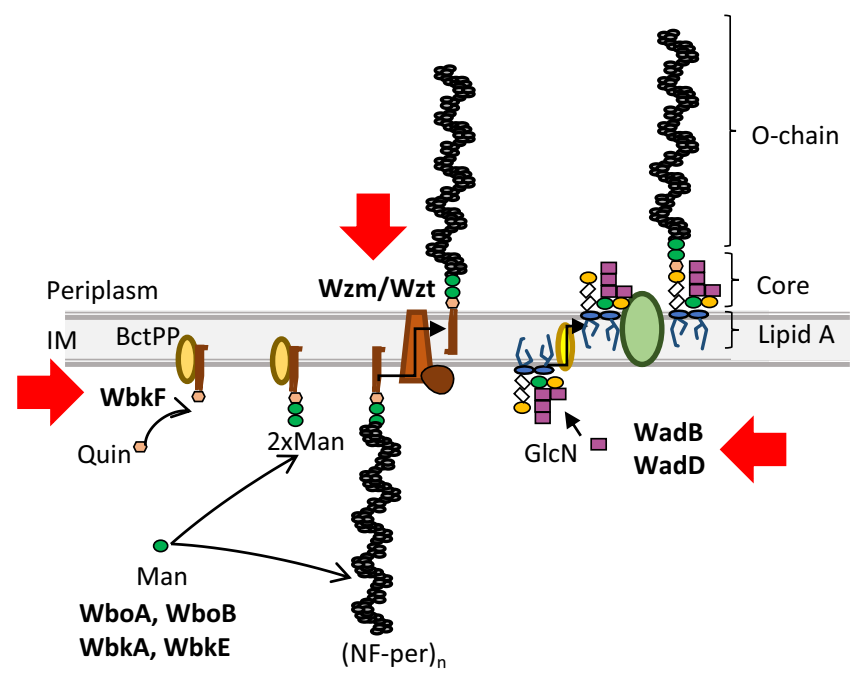

B

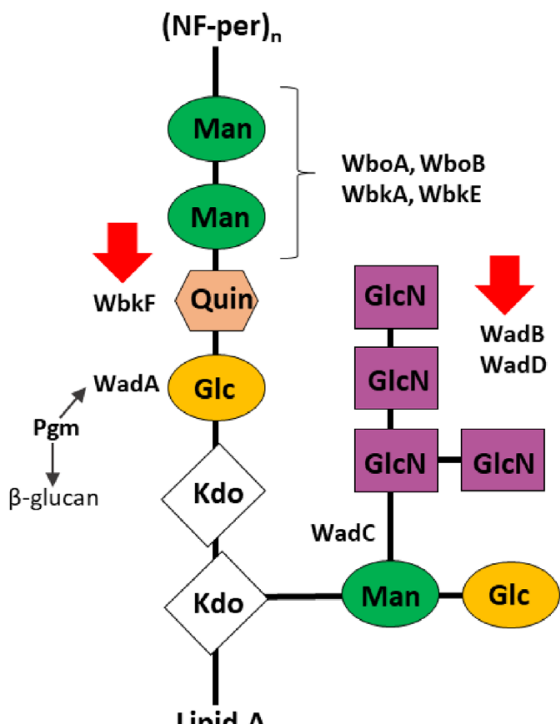

C

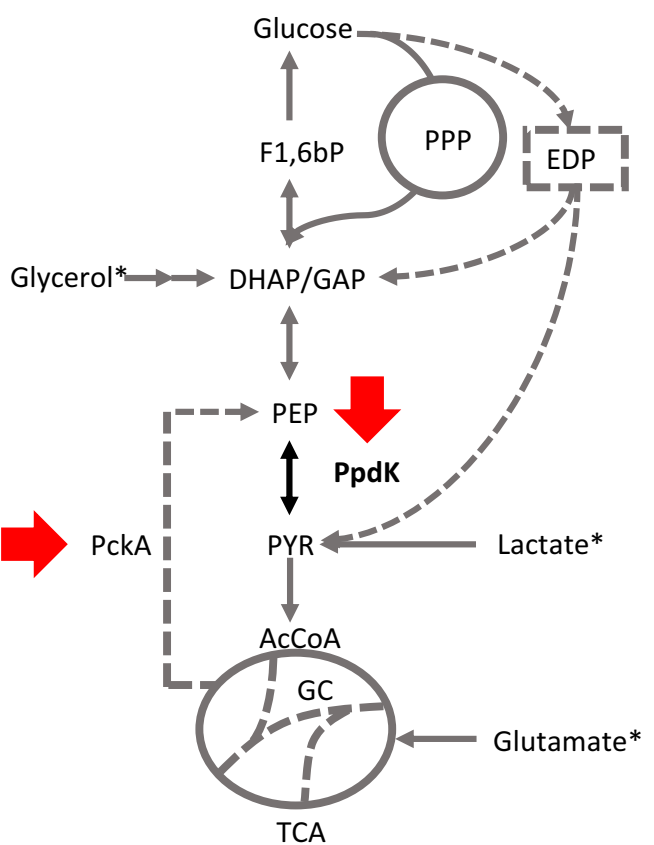

Figure 1 Pathways and key enzymes targeted for the development of B. suis vaccines. Schematic representation of Brucella (A) LPS biosynthesis steps that occur at both sides of the inner membrane (IM), B LPS core, and $\mathbf{C}$ central carbon metabolism. The proteins corresponding to the mutants investigated are indicated with arrows. WbkF, bactoprenol (BctPP, Bactoprenol-P-P) primase for O-chain polymerization; Wzm/ Wzt, O-chain translocation ABC transport system; WadA, core glycosyltransferase that incorporates the terminal glucose (Glc) linking the core to the two O-chain mannoses (Man) of the proximal section of the N-formyl-perosamine (NF-per) polysaccharide; Pgm, phosphoglucomutase necessary for the synthesis of the UPD-glucose used by WadA; WadB, WadC and WadD, glycosyltransferases involved in the incorporation of glucosamine (GlcN) and Man to the core-lateral branch; Kdo, 3-deoxy-D-manno-octulosonic acid; Quin, quinovosamine. WboA, WboB, WbkA and WbkE, O-chain glycosyltransferases. F1,6bP, fructose-1,6-bisphosphate; DHAP, dihydroxyacetone-phosphate; GAP, glyceraldehyde-3-phosphate; PEP, phosphoenolpyruvate; PYR, pyruvate; AcCoA, acetyl-COA; PckA, phosphoenolpyruvate carboxykinase; PpdK, pyruvate phosphate dikinase; EDP, Entner-Doudoroff pathway; PPP, pentose phosphate pathway; TCA, tricarboxylic acid cycle; GC, glyoxylate bypass. Asterisks mark substrates in the mGSM. The functionality of pathways represented as discontinuous lines varies depending on the Brucella species and biovar [17, 29-34, 56]. 
production and enhance the immune response (Table 1) [17, 18, 28-31]. Moreover, no attempts to develop an attenuated $B$. suis vaccine have been made by exploiting our current understanding of Brucella metabolism [32], and here we also investigated $B$. suis bv2 vaccines lacking pyruvate phosphate dikinase (PpdK) and/or phosphoenolpyruvate carboxykinase (PckA) activities (Table 1 and Figure 1C) [33, 34]. We conducted our research in a $B$. suis bv2 strain because, since this biovar is not zoonotic, it is particularly appropriate for developing a vaccine posing no risks for humans.

\section{Materials and methods}

\section{Bacterial strains and growth conditions}

The bacterial strains and plasmids used are listed in Additional file 1. We used B. suis bv2 CITA 198 (herein Bs2WT) because, although Bs2WT and the B. suis bv2 reference strain (B. suis bv2 Thomsen) have the same PCR-RFLP pattern [3], the former shows a virulence pattern in mice typical of $B$. suis bv2 field strains and the latter is attenuated (Additional file 2).

Bacteria were routinely grown either in standard tryptic soy broth (TSB; Scharlau, Barcelona, Spain) or TSA (TSB supplemented with agar [Pronadisa, Laboratorios Conda, Spain]) at $37^{\circ} \mathrm{C}$. For the studies in mice, vaccines and challenge strains were grown on Blood Agar Base (BAB; Oxoid, UK). When needed, media were supplemented with $5 \%$ sucrose, diaminopimelic acid (DAP [Sigma]; $1 \mathrm{mM}$ ), 0.2\% activated charcoal, kanamycin $(\mathrm{Km})$ at $50 \mu \mathrm{g} / \mathrm{mL}$ or at $35 \mu \mathrm{g} / \mathrm{mL}$, ampicillin (Amp) at $100 \mu \mathrm{g} / \mathrm{mL}$ and/or chloramphenicol (Cm) at $20 \mu \mathrm{g} / \mathrm{mL}$ (all from Sigma). The lactate-glutamateglycerol-vitamins synthetic medium of Gerhardt's [35] was supplemented with $1 \mathrm{mM}$ methionine (mGSM) (this amino acid is required for growth of some Brucella strains in synthetic media [32] including Bs2WT (Zúñiga-Ripa, unpublished observations). All strains were stored at $-80{ }^{\circ} \mathrm{C}$ in skimmed milk (Scharlau, Barcelona, Spain) or in TSB supplemented with $0.5 \%$ yeast extract (Pronadisa, Laboratorios Conda, Spain) (TYSB) and $7 \%$ dimethylsulfoxide.

\section{Sequence analyses and DNA manipulation}

Genomic sequences of $B$. suis bv2 Thomsen (ATCC 23445 or NCBI:txid470137), B. melitensis 16M (ATCC 23456 or NCBI:txid224914) and B. abortus 2308 (NCBI:txid359391) were obtained from databases at the National Center for Biotechnology Information (NCBI) or Kyoto Encyclopedia of Genes and Genomes (KEGG: https://www.genome.jp/kegg/). Since the genomic sequence of Bs2WT was not available, the ORFs of interest were PCR amplified and sequenced by "Servicio de Secuenciación del Centro de Investigación Médica Aplicada" (CIMA, Pamplona, Spain). Sequence alignments were performed with Clustal Omega (https ://www.ebi.ac.uk/Tools/msa/clustalo/).

Plasmids and chromosomal DNA were extracted with QIAprep ${ }^{\circledR}$ Spin Miniprep Kit and QIAamp ${ }^{\circledR}$ DNA Miniprep Kit (Qiagen GmbH, Hilden, Germany). When needed, DNA was purified from agarose gels using a QIAquick ${ }^{\circledR}$ Gel Extraction Kit (Qiagen GmbH, Hilden, Germany). Primers were designed using Primer 3 input (http://primer3.ut.ee/) and synthesized by SigmaGenosys Ltd (Haverhill, United Kingdom).

Table 1 Genes studied in the present work.

\begin{tabular}{|c|c|c|c|c|}
\hline Gene & Coding for & LPS phenotype & Comments & References \\
\hline wadB & $\begin{array}{l}\text { Glycosyltransferase involved in the synthesis of } \\
\text { Brucella LPS core lateral branch }\end{array}$ & S-LPS with core defect & $\begin{array}{l}\text { B. abortus mutants shown to be attenuated in } \\
\text { mice }\end{array}$ & {$[30,31]$} \\
\hline wadD & $\begin{array}{l}\text { Glycosyltransferase involved in the synthesis of } \\
\text { Brucella LPS core lateral branch }\end{array}$ & S-LPS with core defect & $\begin{array}{l}\text { B. abortus mutants shown to be attenuated in } \\
\text { mice }\end{array}$ & {$[29]$} \\
\hline$w b k F$ & $\begin{array}{l}\text { Undecaprenyl-glycosyltransferase priming bacto- } \\
\text { prenol for O-chain polymerization }\end{array}$ & R-LPS with complete core & $\begin{array}{l}\text { B. melitensis mutants shown to be attenuated in } \\
\text { mice }\end{array}$ & {$[17]$} \\
\hline$w z m$ & $\begin{array}{l}\text { Permease of the } A B C \text { system translocating the } \\
\text { O-chain to the periplasm }\end{array}$ & R-LPS with complete core & $\begin{array}{l}\text { Build up cytoplasmic O-chain on the inner } \\
\text { membrane. B. melitensis mutants shown to be } \\
\text { attenuated in mice and to trigger antibodies to } \\
\text { the O-chain }\end{array}$ & {$[17,55]$} \\
\hline$p p d K$ & Pyruvate phosphate dikinase (PEP $\leftrightarrow$ pyruvate) & S-LPS & $\begin{array}{l}\text { Required for growth on gluconeogenic media } B \text {. } \\
\text { abortus mutants are attenuated in mice }\end{array}$ & {$[33,34]$} \\
\hline$p c k A$ & $\begin{array}{l}\text { Phosphoenolpyruvate carboxykinase (oxaloac- } \\
\text { etate } \rightarrow \text { PEP) }\end{array}$ & S-LPS & $\begin{array}{l}\text { Inactive in } B \text {. abortus but not in B. suis biovar } 5 \text {. } \\
\text { Together with ppdK is required for growth of } \\
B \text {. suis biovar } 5 \text { in gluconeogenic media. } B \text {. suis } \\
\text { biovar } 5 \text { mutated in both ppdK and pckA are } \\
\text { attenuated in mice }\end{array}$ & {$[33,34]$} \\
\hline
\end{tabular}




\section{Mutant construction}

We obtained Bs $2 \Delta w a d B$, Bs $2 \Delta w a d D$, Bs $2 \Delta p p d K$, Bs2 $2 p p d K \Delta p c k A, \quad$ Bs2 $\Delta p p d K \Delta w a d B \quad$ and Bs2 $2 p p d K \Delta w a d D$ (all with O-chain) and Bs $2 \Delta w b k F$ and $\mathrm{Bs} 2 \Delta w z m$ (R; O-chain defective) by in-frame deletion of the corresponding genes (Table 1 and Additional file 1). For the construction of Bs $2 \Delta w a d B$, Bs $2 \Delta w a d D$ and $\mathrm{Bs} 2 \Delta p p d K$, we used the suicide plasmids previously shown to generate in-frame deletions in $B$. abortus (the sequences where oligonucleotides hybridize are identical in Bs2WT) [29, 30, 33, 34]. To this end, the plasmids were extracted from E. coli TOP10F' and transformed into $E$. coli $\beta 2150$, a diaminopimelic acid (DAP) auxotrophic donor strain [36]. For Bs $2 \Delta w a d B$ construction, the suicide plasmid pJQK $\Delta w a d B$ [30] was introduced in Bs2WT by tri-parental mating with conjugative $E$. coli B2150-pJQK $\Delta w a d B$ and E. coli $\beta 2150$-pRK2013 as helper strain [37]. The first recombination event was selected by $\mathrm{Km}$ resistance and DAP independence and confirmed by PCR. The allelic exchange by double recombination was selected for growth on sucrose and $\mathrm{Km}$ sensitivity. The same strategy was followed to construct Bs $2 \Delta w a d D$ and Bs2 $\Delta p p d K$ using suicide plasmids pJQK $\Delta w a d D$ [29] and pJQK $\Delta p p d K$ [34]. The double mutants Bs2 $\Delta p p d K \Delta w a d B$ and Bs $2 \Delta p p d K \Delta w a d D$ were constructed by deletion of $w a d B$ or $w a d D$ in Bs $2 \Delta p p d K$ as described above. The double mutant Bs $2 \Delta p p d K \Delta p c k A$ was constructed by deleting $p c k A$ in $\mathrm{Bs} 2 \Delta p p d K$ using plasmid pJQK $\Delta p c k A$ [34]. The loss of the plasmid concomitant with the gene deletion in each of these mutants was confirmed by PCR with the corresponding oligonucleotides (see Additional file 3).

For the construction of Bs $2 \Delta w z m$, we first generated two PCR fragments: oligonucleotides $w z m-\mathrm{F} 1$ and $w z m$ R2 (Additional file 4) were used to amplify a 484 bp fragment including codons 1 to 31 of BMEI1415, as well as 390 bp upstream of the start codon. Oligonucleotides $w z m-\mathrm{F} 3$ and $w z m-\mathrm{R} 4$ (Additional file 4) were used to amplify a 447 bp fragment including codons 247 to 265 of BMEI1415 and 385 bp downstream of the stop codon. These fragments were ligated by overlapping PCR using $w z m-\mathrm{F} 1$ and $w z m-\mathrm{R} 4$ for amplification, and the complementary regions between R2 and F3 for overlapping. The resulting sequence, containing the $w z m$ deletion allele, was cloned into pCR2.1 (Invitrogen) to generate plasmid pCR2.1 $\Delta w z m$, sequenced to ensure the maintenance of the reading frame, subcloned into the BamHI and XbaI sites of the suicide plasmid PJQK [38] and transformed into competent $E$. coli $\beta 2150$. The resulting suicide pJQK-derived plasmid (pJQK $\Delta w z m$ ) was introduced in Bs2WT by conjugation following the procedure described above. Bs $2 \Delta w b k F$ was constructed following a similar strategy using oligonucleotides $w b k F-F 1$ and
wbkF-R2 (Additional file 4) that amplified a $448 \mathrm{bp}$ fragment (including codons 1 to 19 of BMEI1426 as well as $390 \mathrm{bp}$ upstream of the BMEI1426 start codon) and oligonucleotides $w b k F-F 3$ and $w b k F-R 4$ (Additional file 4) that amplified a $505 \mathrm{bp}$ fragment (including codons 301 to 335 of BMEI1426 and 398 bp downstream of the BMEI1426 stop codon). Both fragments were ligated, cloned into pCR2.1 to generate plasmid pCR2.1 $\Delta w b k F$, subcloned into $\mathrm{pJQK}(\mathrm{pJQK} \Delta w b k F)$ and transformed into competent $E$. coli $\beta 2150$. After conjugation with Bs $2 \mathrm{WT}$, we performed PCRs (primers are described in Additional file 3) to screen the resulting colonies for the $w b k F$ or wzm deletion.

We constructed a kanamycin resistant challenge strain $\left(\mathrm{Bs} 2:: \operatorname{Tn} 7 \mathrm{Km}^{\mathrm{R}}\right.$ ) to discriminate the challenge and vaccine strains in the mouse protection experiments (see below). For this, we inserted the pUC18R6KT-miniTn7Km vector into the Bs2WT chromosome by tetra-parental conjugation and selected the conjugants harboring this modified miniTn7 by plating onto TSA-DAP-Km. The construct $\left(\mathrm{Bs} 2:: \operatorname{Tn} 7 \mathrm{Km}^{\mathrm{R}}\right)$ was examined by PCR [39] for the correct insertion and orientation of the transposon between the $g \operatorname{lm} S$ and $r e c G$ genes $[40,41]$.

\section{Bacteriological typing}

All strains were typed following established Brucella procedures [2]: i.e. colonial morphology, urease, agglutination with anti-A and anti-M monospecific sera recognizing the cognate epitopes in the O-chain of Brucella S-LPS, susceptibility to thionine, fuchsine and safranin dyes and sensitivity to $\mathrm{Tb}, \mathrm{Wb}, \mathrm{Iz}$ and $\mathrm{R} / \mathrm{C}$ phages. $\mathrm{S} / \mathrm{R}$ colony morphology was studied by the crystal violet dye exclusion test and by acriflavine agglutination [2].

\section{Growth curves}

To obtain inocula preconditioned for growth in each test medium (TSB or mGSM), bacteria were first grown in $10 \mathrm{~mL}$ of TSB in a $50 \mathrm{~mL}$ flask at $37^{\circ} \mathrm{C}$ with orbital shaking for $18 \mathrm{~h}$. In the case of TSB growth curves, these exponentially growing bacteria were harvested by centrifugation, resuspended at an O.D. ${ }_{600 \mathrm{~nm}}$ of 0.1 in the same medium and $200 \mu \mathrm{L} /$ well aliquots were dispensed by triplicate in Bioscreen multi-well plates. In the case of mGSM growth curves, the exponentially growing bacteria in TSB were harvested by centrifugation, resuspended in $10 \mathrm{~mL}$ of mGSM at an optical density at $600 \mathrm{~nm}$ (O.D. ${ }_{600 \mathrm{~nm}}$ ) of 0.1 , and incubated at $37{ }^{\circ} \mathrm{C}$ with orbital shaking for $18 \mathrm{~h}$. These preconditioned bacteria were harvested by centrifugation, resuspended at an O.D. ${ }_{600 \mathrm{~nm}}$ of 0.1 in the same medium and $200 \mu \mathrm{L} /$ well aliquots were dispensed by triplicate in Bioscreen multi-well plates. The plates were cultivated in a Bioscreen C (Lab Systems) apparatus with continuous shaking at $37^{\circ} \mathrm{C}$. Growth was 
monitored spectrophotometrically at $420-580 \mathrm{~nm}$ every $30 \mathrm{~min}$ over a $60 \mathrm{~h}$-period. All experiments were performed in triplicate (biological replicates). Wells containing sterile medium were used as negative controls in all experiments.

\section{LPS characterization}

LPS from Bs2WT and mutants in LPS genes (Table 1) were extracted by the proteinase K sodium dodecyl sulfate (SDS) protocol [42], an extraction procedure valid for S and R Brucella [17, 43]. Briefly, phenol-inactivated bacteria (grown in TSB) were centrifuged $(20 \mathrm{~min}$ at $6500 \times g)$ and washed with saline. The cells were suspended in $2 \%$ SDS-60 mM Tris- $\mathrm{HCl}$ buffer ( $\mathrm{pH} 6.8$ ), heated at $100{ }^{\circ} \mathrm{C}$ for $10 \mathrm{~min}$ and treated with proteinase $\mathrm{K}(60 \mu \mathrm{L}$ of a $2.5 \mathrm{mg} / \mathrm{mL}$ stock in Tris- $\mathrm{HCl}$ per each $\mathrm{mL}$ of suspension) for $3 \mathrm{~h}$ at $55{ }^{\circ} \mathrm{C}$ and overnight at room temperature. Then, samples were centrifuged $(30 \mathrm{~min}$ at $20000 \times g$ ) and the supernatants precipitated with 3 volumes of methanol containing $1 \%$ sodium acetate-saturated methanol at $-20{ }^{\circ} \mathrm{C}$ for $60 \mathrm{~min}$. The precipitates were collected by centrifugation $(15 \mathrm{~min}$ at $5000 \times g)$, and resuspended in $10 \mathrm{~mL}$ of distilled water, precipitated again with methanol and sedimented again by centrifugation. The pellets were resuspended in $3 \mathrm{~mL}$ of $60 \mathrm{mM}$ Tris- $\mathrm{HCl}(\mathrm{pH} 6.8)$, digested once with nucleases $[20 \mu \mathrm{L} /$ $\mathrm{mL}$ each of DNase and RNase stock solutions at $0.5 \mathrm{mg} /$ $\mathrm{mL}$ in Tris-HCl (MP Biomedicals and Sigma-Aldrich, respectively)] at $37^{\circ} \mathrm{C}$ for $30 \mathrm{~min}$ and treated again with proteinase $\mathrm{K}\left(60 \mu \mathrm{L} / \mathrm{mL}, 3 \mathrm{~h}\right.$ at $\left.55^{\circ} \mathrm{C}\right)$. Finally, the LPS were harvested by centrifugation $(15 \mathrm{~min}$ at $5000 \times \mathrm{g})$, suspended in $1 \mathrm{~mL}$ of distilled water and stored at $-20{ }^{\circ} \mathrm{C}$.

For LPS characterization, LPS samples were analyzed in $15 \%$ polyacrylamide gels (37.5:1 acrylamide/methylenebisacrylamide ratio) in Tris-HCl-glycine and revealed by the periodate-alkaline silver method [44]. For western blots, gels were electro-transferred onto nitrocellulose sheets (Amersham-GE Healthcare Life Scientific, Germany; $0.45 \mu \mathrm{m}$ pore) using $25 \mathrm{mM}$ Tris, $192 \mathrm{mM}$ glycine ( $\mathrm{pH} 8.3)$ and $20 \%(\mathrm{vol} / \mathrm{vol})$ methanol in a Trans-Blot Semi-Dry Transfer Cell (Bio-Rad) at a constant voltage of $8 \mathrm{~V}$ and $200 \mathrm{~mA}$ for $30 \mathrm{~min}$. Membranes were blocked with $5 \%$ skimmed milk in phosphate buffered saline (PBS; $10 \mathrm{mM}$ phosphate $140 \mathrm{mM} \mathrm{NaCl}, 2.7 \mathrm{mM} \mathrm{KCl}$ [pH 7.4]; Medicago, Sweden) with $0.05 \%$ Tween 20 (PBS-T) overnight at $4{ }^{\circ} \mathrm{C}$, and then washed with the same buffer. Membranes were then incubated with primary antibody diluted 1:500 in PBS-T overnight at room temperature, washed with PBS-T and bound immunoglobulins were detected with peroxidase labeled protein $\mathrm{G}$ and 4-chlorine-1-naphtol- $\mathrm{H}_{2} \mathrm{O}_{2}$. Monoclonal antibody A68-24G12/ A08 from the ascitic fluid of an infected mouse, which recognizes core epitopes [45], and a serum of a rabbit infected with $B$. melitensis $16 \mathrm{M}$ and bled 45 days later were used.

\section{Mouse experiments}

Seven-week-old female BALB/c mice (ENVIGO, Harlan) were lodged in cages in a BSL-3 facility (ES/31-2010000132) with water and food ad libitum for 2 weeks before and during the experiments in accordance with the current European (directive 86/609/EEC) and Spanish (RD 53/2013) legislations.

For virulence assessment, groups of 5 mice per time-point were inoculated with each mutant (Bs $2 \Delta w a d B, B s 2 \Delta w a d D$, Bs $2 \Delta p p d K$, Bs $2 \Delta p p d K \Delta w a d B$, $\mathrm{Bs} 2 \Delta p p d K \Delta w a d D, \mathrm{Bs} 2 \Delta w z m$ and $\mathrm{Bs} 2 \Delta w b k F)$ or Bs2WT. Inocula were prepared harvesting $B A B$ grown bacteria in sterile buffered saline (BSS; $0.85 \% \mathrm{NaCl}, 0.1 \%$ $\mathrm{KH}_{2} \mathrm{PO}_{4}, 0.2 \% \mathrm{~K}_{2} \mathrm{HPO}_{4}$; $\left.\mathrm{pH} 6.85\right)$ and adjusting the bacterial suspension to $1 \times 10^{9} \mathrm{CFU} / \mathrm{mL}$ (inoculum used for $1 \times 10^{8} \mathrm{CFU}$ doses in the immunization with $\mathrm{R}$ vaccines). This suspension was then diluted to $1 \times 10^{6} \mathrm{CFU} / \mathrm{mL}$ to prepare the inocula used for $\mathrm{S}$ vaccines $\left(1 \times 10^{5} \mathrm{CFU}\right.$ doses). Mice were inoculated intraperitoneally (IP) with $0.1 \mathrm{~mL}$ of each inoculum and the exact dose was determined retrospectively on triplicate BAB plates. At several intervals after inoculation, mice were euthanized, the spleens were aseptically removed, individually weighed, homogenized in 9 volumes of BSS and serial tenfold dilutions plated by triplicate on BAB plates. After 5 days at $37^{\circ} \mathrm{C}$, CFU were counted, colonies checked by the crystal violet exclusion test and the identity confirmed by PCR. The data (mean CFU/spleen) were normalized by logarithmic transformation, and mean $\log _{10} \mathrm{CFU} /$ spleen values and standard deviations (SD) calculated for plotting and statistical comparison by one-way ANOVA and Fisher's Protected Least Significant Differences (PLSD) tests.

To determine the protective efficacy, groups of 5 $\mathrm{BALB} / \mathrm{c}$ mice were inoculated subcutaneously (SC) with $10^{5} \mathrm{CFU} /$ mouse of Bs $2 \Delta p p d K$, Bs $2 \Delta w a d B$ and Bs $2 \Delta p p d K \Delta w a d B$ or $10^{8} \mathrm{CFU} /$ mouse of Bs $2 \Delta w b k F$ and Bs2 $\Delta w z m$. The Rev1 reference vaccine $\left(10^{5} \mathrm{CFU} /\right.$ mouse $)$ or BSS inoculated mice were used as effective-vaccine and unvaccinated controls, respectively. Four weeks after vaccination, all animals were challenged IP with $10^{5} \mathrm{CFU}$ of the virulent $\mathrm{Bs} 2:: \operatorname{Tn} 7 \mathrm{Km}^{\mathrm{R}}$ strain. After 2 weeks, CFU/ spleen numbers of the challenge strain were determined by plating on $B A B \mathrm{Km}$. Values of residual vaccine were also calculated by subtracting CFU numbers on $\mathrm{BAB}$ $\mathrm{Km}$ to those obtained on BAB. Results were expressed as the mean $\log _{10} C F U /$ spleen $\pm S D(n=5)$ and statistical comparisons between vaccines and controls for challenge strain values were made using ANOVA and PLSD tests. The virulence of the $\mathrm{Bs} 2:: \mathrm{Tn} 7 \mathrm{Km}^{\mathrm{R}}$ challenge strain 
used in this work was verified in a previous experiment in BALB/c mice $(n=5)$ by IP infection $\left(10^{5} \mathrm{CFU} /\right.$ mouse $)$ and CFU/spleen counting 2 and 6 weeks later. This strain showed identical CFU in BAB-S and BAB-Km, and virulence pattern identical to that of Bs2WT (not shown).

\section{Results}

\section{Construction and characterization of B. suis biovar 2 LPS mutants}

We first confirmed the presence of the orthologues of $B$. abortus wadB and wadD in B. suis bv2 using the complete sequence of the genome of $B$. suis bv2 Thomsen available in databases. Then, as this strain was not suitable for determining the actual degree of attenuation of the mutants (see "Materials and methods" and Additional file 2), we amplified and sequenced the corresponding regions in the genome of Bs2WT, a virulent strain (Additional file 2). We found that these two ORFs were highly conserved in Bs2WT and, accordingly, we obtained the non-polar mutants Bs $2 \Delta w a d B$ and Bs $2 \Delta$ wadD using the tools developed before for $B$. abortus (see Table 1 and Additional file 1).

Bs $2 \Delta w a d B$ and Bs $2 \Delta w a d D$ were identical to the Bs2WT parental strain in growth rates in TSB and mGSM (data not shown) as well as in urease production, sensitivity to thionine, fuchsine and safranin dyes, and in agglutination with the monospecific A but not with the monospecific $M$ sera (Additional file 5). This last result indicates that the $\mathrm{O}$-chain is conserved in the mutants as predicted by the function assigned to WadB and WadD (Table 1 and Figure $1 \mathrm{~A}-\mathrm{B}$ ), and this was also confirmed by western blot analysis (Figure 2A). Analysis of the core region showed the predicted (Table 1) small molecular weight shift (Figure 2B) indicative of the lack of some sugars in the core lateral branch (Figure 1B) and the subsequent defect in the core epitope recognized by monoclonal A68-24G12/A08 (Figure 2C). Altogether, these results show that $\mathrm{Bs} 2 \Delta w a d B$ and $B s 2 \Delta w a d D$ are homologous in LPS structure to their $B$. abortus counterparts $[29,30]$.

Following the same genomic approach, we also constructed R-LPS mutants. First, we identified the O-chain primase $(w b k F)$ and O-chain translocation $(w z m)$ genes (Table 1) in B. suis bv2 Thomsen and then we confirmed by amplification and sequence analysis that they were conserved in Bs2WT. Based on this, we constructed the corresponding in frame-deletion mutants and confirmed the $\mathrm{R}$ phenotype by a negative result in the crystal violet exclusion test, a positive acriflavine agglutination and no agglutination with the monospecific anti-A and anti-M sera (that recognize $\mathrm{O}$-chain epitopes with variable dominance depending on the S Brucella species and biovar). Although both mutants were resistant to phage

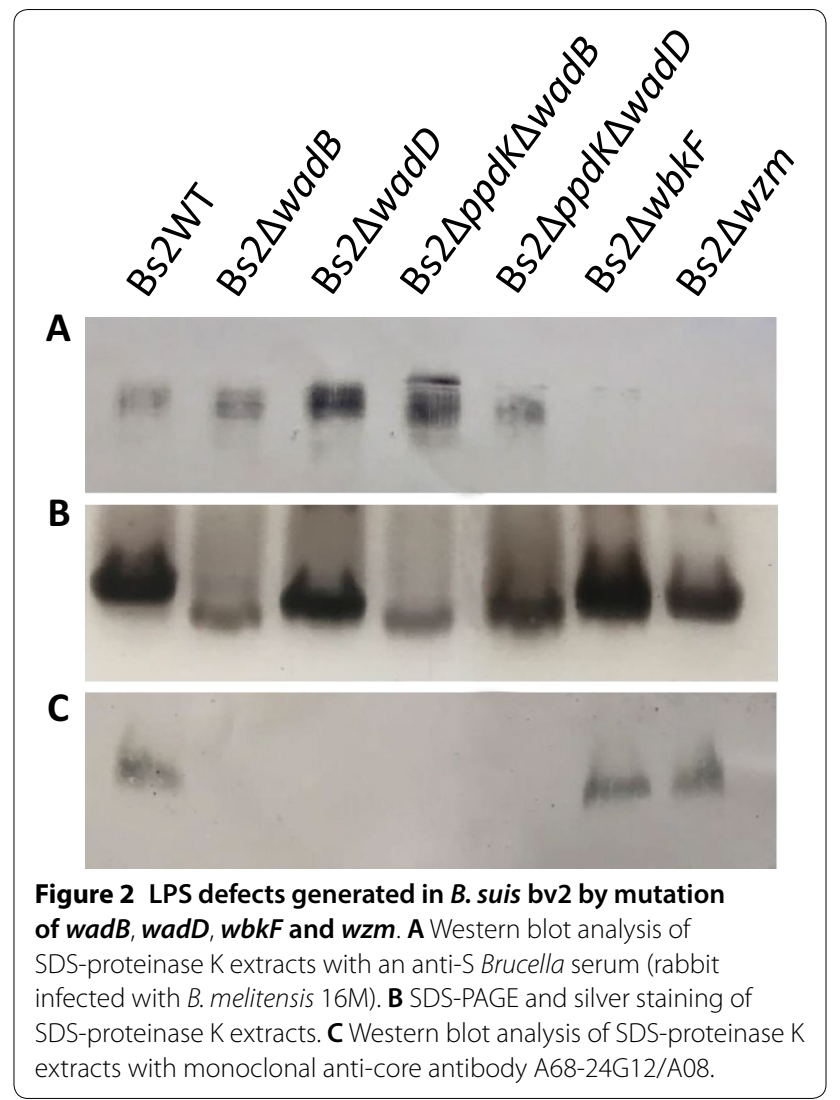

$\mathrm{R} / \mathrm{C}$ (Additional file 5), this was not totally unexpected because this phage (originally obtained from a mixture of S specific brucella phages by $N$-methyl- $N^{\prime}$-nitro- $N$ nitrosoguanidine treatment and serial propagation on B. abortus, B. ovis and B. canis) is known to display variable activity depending upon the Brucella species origin of the $\mathrm{R}$ mutant [46]. Further analyses by SDS-PAGE and western blot showed that these mutants carried a LPS that lacked reactivity with antibodies recognizing $\mathrm{O}$-chain epitopes and that, in contrast to $\mathrm{Bs} 2 \Delta$ wadB and $B s 2 \Delta w a d D$, displayed mobility and anticore antibody reactivity similar to those of the R-LPS fraction of Bs2WT (Figure 2A-C). Therefore, these mutants carry a R-LPS with a complete core, as predicted (Table 1).

\section{Construction and characterization of $B$. suis biovar 2 metabolic mutants}

First, we searched for orthologues of $p p d K$ and $p c k A$ (Table 1 ) in the $B$. suis bv2 Thomsen genome. We found that Thomsen $p p d K$ is highly conserved with respect to B. abortus 2308 and B. suis 513 (biovar 5), two strains where its functionality has been shown (Table 1). Moreover, sequencing of the homologous region confirmed its conservation in Bs2WT. Therefore, we constructed a Bs $2 \Delta p p d K$ in-frame deletion 
mutant of Bs2WT and compared its growth in TSB and mGSM. As can be seen in Figure 3, deletion of $p p d K$ abrogated growth in the latter medium but not in TSB, indicating that in $3 \mathrm{C}$ and $4 \mathrm{C}$ substrates the PEP (phosphoenolpyruvate) (and growth in gluconeogenic conditions) depends on PpdK (Figure 1C). Although in $B$. suis biovar 5, PEP [33] can result also from the activity of PckA in gluconeogenic medium (Figure 1C), this observation suggested us that PckA is not functional in Bs2WT, a phenotype we already described for B. abortus [34]. In fact, the complete sequence of B. suis bv2 Thomsen shows a premature stop codon in $p c k A$ (Figure 4) not present in B. suis biovar 5 or B. microti [33]. A premature stop codon also occurs in pckA in all $B$. abortus and $B$. melitensis sequenced strains. However, while in these species the stop codon is located at position 1474-1476, in B. suis bv2 Thomsen it is located at position 492-494 (Figure 4 and Additional file 6). Upon sequencing, we observed that the Bs2WT also displayed a truncated version of $p c k A$ that interestingly resulted from a deletion at position 217 generating a premature stop codon at position 254-256, thus different from those in B. abortus, B. melitensis or $B$. suis bv2 Thomsen (Figure 4). Remarkably, a database search in KEGG showed that the same mutation found in B. suis bv2 Thomsen is present in the genome of all $B$. suis bv2 strains sequenced thus far. Indeed, a double mutant Bs2 $\Delta p p d K \Delta p c k A$ showed the same inability to grow on mGSM as Bs $2 \Delta p p d K$ (Figure 3 ). These findings strongly suggest that the lack of activity of PckA observed in B. abortus [33] is extended to at least $B$. suis bv2.

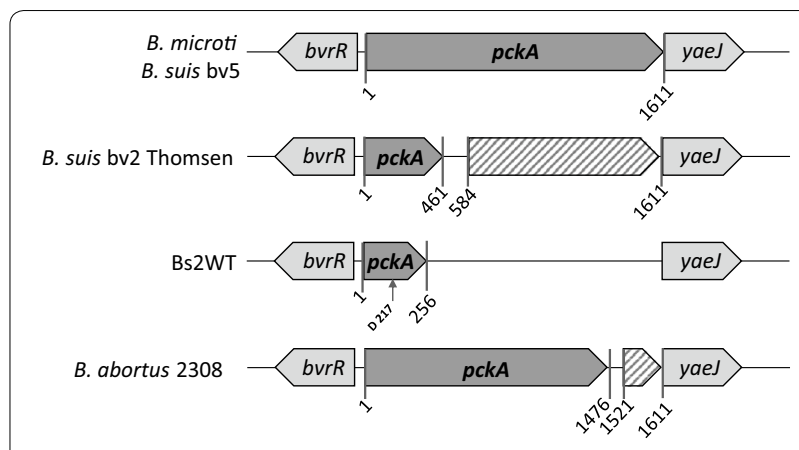

Figure 4 B. suis bv2 presents a SNP leading to a premature stop in $\boldsymbol{p c k \boldsymbol { A }}$. The sites where premature stop codons occur are indicated with a vertical arrow. The SNP leading to premature stop in Bs2WT is indicated as $D$ (for deletion). The functional $p c k A$ of B. microti and $B$. suis biovar 5 , and the mutated $p c k A$ of $B$. abortus described previously are included as reference. In all brucellae, $p c k A$ is located upstream of the regulatory gene bvrR. In Brucella presenting an intact pckA, yaeJ (coding a ribosome-associated protein) is located immediately downstream of $p c k A$, whereas in the sequenced Brucella presenting a mutated $p c k A$ a ORF (i.e.: a truncated PckA in B. abortus or a pseudogene in $B$. suis bv2 Thomsen) upstream of yaeJ is annotated (stripped arrows).

\section{B. suis vaccine candidates display different profiles of attenuation in mice}

We evaluated the colonization and persistence of Bs2WT mutants in the spleen of mice by IP inoculation with $1 \times 10^{8}$ and $1 \times 10^{5} \mathrm{CFU} /$ mouse of mutants with $\mathrm{R}$ and $\mathrm{S}$ LPS, respectively (Figure 5). The CFU/spleen values of all LPS mutants were similar to those of Bs2WT at post-infection week 2 . However, at post-infection week 6 , the CFU/spleen of all these mutants were significantly lower $(\mathrm{p}<0.005)$ than those of Bs2WT but with different

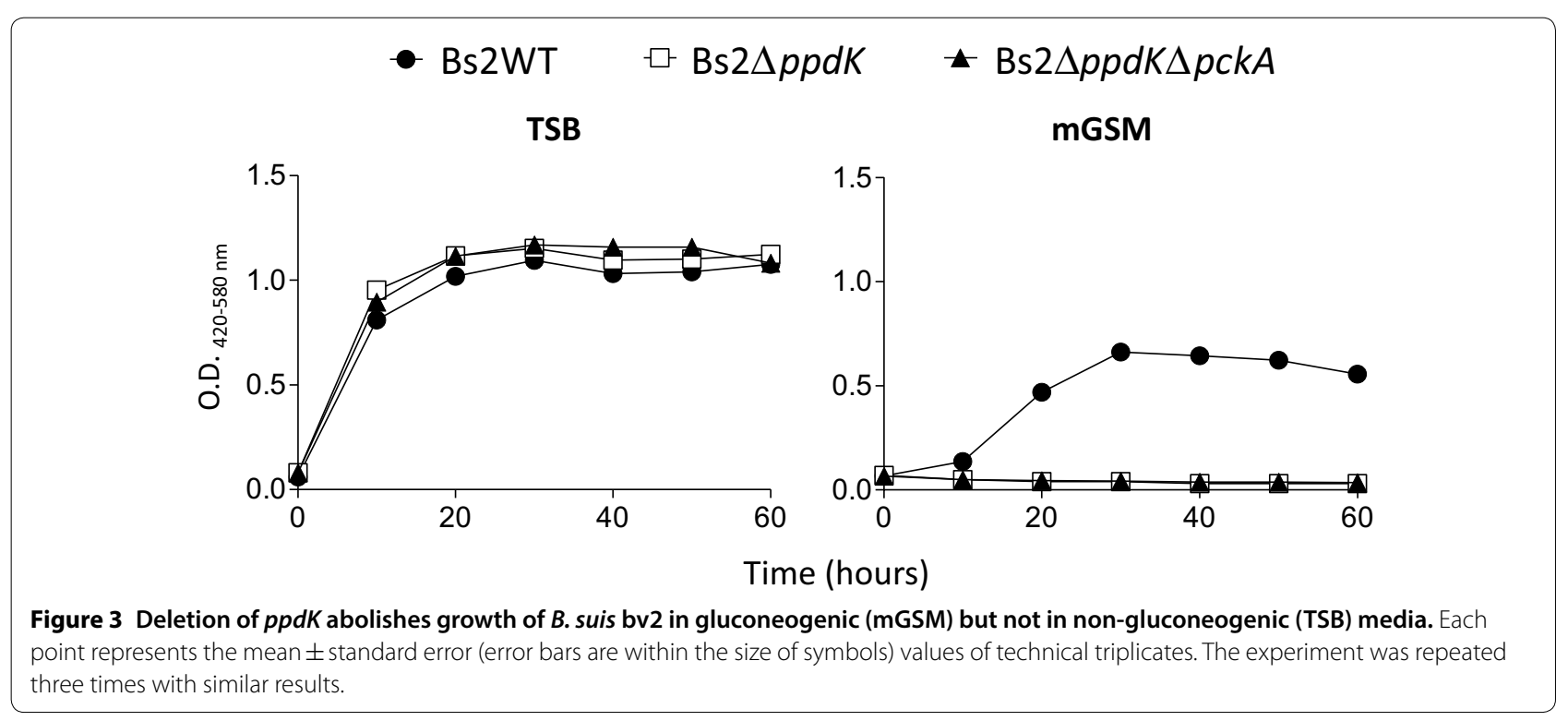


A LPS mutants

- Bs2WT

$\triangle \mathrm{Bs} 2 \Delta w a d B \quad \mathrm{Bs} 2 \Delta w b k F$

$\diamond \mathrm{Bs} 2 \Delta w a d D=\mathrm{Bs} 2 \Delta w z m$
B Metabolic mutants

Bs2WT

$\square \mathrm{Bs} 2 \Delta p p d K$
C Combined mutants

- Bs2WT

$\neg$ Bs2 $2 p p d K \Delta w a d B$

- $\mathrm{Bs} 2 \Delta p p d K \Delta w a d D$
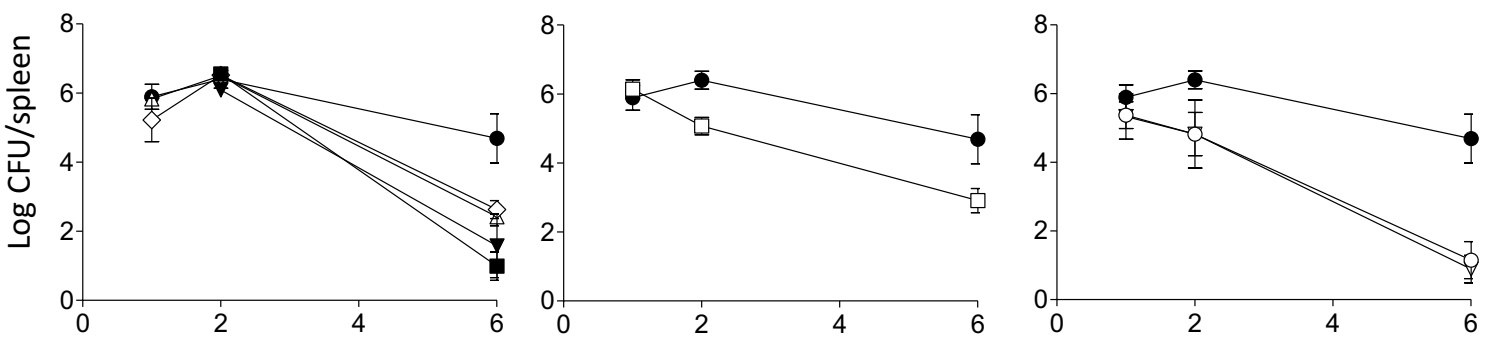

Time post infection (weeks)

Figure 5 B. suis vaccine candidates display different profiles of multiplication in mouse spleen. Multiplication profile of A LPS mutants, B ppdK metabolic mutant and $\mathbf{C}$ combined mutants. CFUs in spleen of infected BALB/C mice were counted after SC inoculation with $10^{5} \mathrm{CFU} / \mathrm{mouse}$ of $\mathrm{Bs} 2 \Delta p p d K, \mathrm{Bs} 2 \Delta w a d B, \mathrm{Bs} 2 \Delta w a d D, \mathrm{Bs} 2 \Delta p p d K \Delta w a d B \mathrm{Bs} 2 \Delta p p d K \Delta w a d D$, or $10^{8} \mathrm{CFU} / \mathrm{mouse}$ of $\mathrm{Bs} 2 \Delta w b k F$ and $\mathrm{Bs} 2 \Delta w z m$.

degrees of attenuation (Figure $5 \mathrm{~A})$. The Bs $2 \Delta w b k F$ and Bs $2 \Delta w z m \mathrm{R}$ mutants showed numbers of $\mathrm{CFU} /$ spleen significantly lower $(\mathrm{p}<0.05$ and $\mathrm{p}<0.0005)$ than those in the spleens of Bs $2 \Delta w a d B$ or Bs $2 \Delta w a d D$ inoculated mice, which were not different from each other. In contrast to the LPS mutants, attenuation generated by $p p d K$ dysfunction was observed $(\mathrm{p}<0.05)$ already after 2 weeks (Figure 5B). Interestingly, at week 6 the $p p d K$ mutant remained in the spleen at levels higher than those of the $\mathrm{R}$ mutants $(\mathrm{p}<0.005)$ and similar to those of the LPS core mutants Bs $2 \Delta w a d B$ and Bs $2 \Delta w a d D$ (Figure $5 B$ ). At the doses used, all mutants induced more splenomegaly than Bs2WT at the peak of the infection, consistent with a higher stimulation of immunity that could in part account for their attenuation (Additional file 7). The above-summarized differences in attenuation profiles prompted us to construct vaccine candidates by combining core and metabolic defects. We constructed mutants carrying inframe deletions in $p p d K$ and $w a d B$ (Bs2 $\Delta p p d K \Delta w a d \mathrm{~B}$ ) or in $p p d K$ and $w a d D$ (Bs2 $2 p p d K \Delta w a d D)$. Growth in either TSB or mGSM was identical to that obtained with Bs $2 \Delta p p d K$ (not shown). As expected, the double mutants showed a LPS profile similar to those of Bs $2 \Delta w a d B$ and $\mathrm{Bs} 2 \Delta w a d D$ (Figure 2). Like Bs $2 \Delta p p d K$, Bs $2 \Delta p p d K \Delta w a d \mathrm{~B}$ and $\mathrm{Bs} 2 \Delta p p d K \Delta w a d D$ were attenuated $(\mathrm{p}<0.005)$ at post-infection week 2 and more attenuated than the corresponding single mutants $(\mathrm{p}<0.0005)$ at post-infection week 6 (Figure 5C), consistent with an additive effect of the mutations and the multiplication defect observed for the $p p d K$ mutant 2 weeks after infection (Figure 5B). As expected from the lower bacteria load, splenomegaly was reduced as compared to that observed for the single mutants (Additional file 7).

\section{Protection in mice}

Based on the above-summarized results, we selected mutants representing the different multiplication profiles for a protection experiment in mice: Bs $2 \Delta p p d K$, $\mathrm{Bs} 2 \Delta w a d B, \mathrm{Bs} 2 \Delta p p d K \Delta w a d \mathrm{~B}, \mathrm{Bs} 2 \Delta w b k F$ and $\mathrm{Bs} 2 \Delta w z m$. The protection efficacy of these mutants was compared with that conferred by Rev1 and mice inoculated with BSS as a control. As can be seen in Table 2, Rev1 (at $10^{5} \mathrm{CFU} /$ mouse) and the five candidates (at $10^{5}$ or $10^{8} \mathrm{CFU} /$ mouse for $\mathrm{S}$ or R phenotypes, respectively) provided similar levels of protection against Bs2WT.

\section{Discussion}

Whereas vaccines against brucellosis that are effective in cattle, sheep and goats, were developed long ago, this is not the case of a vaccine against swine brucellosis. In part, this may be due to the higher emphasis placed on

Table 2 Protection against virulent B. suis induced by LPS and metabolic mutants inoculated subcutaneously.

\begin{tabular}{llll}
\hline Vaccine & $\begin{array}{l}\text { Dose (CFU/ } \\
\text { mouse) }\end{array}$ & \multicolumn{2}{l}{$\begin{array}{l}\text { Mean } \pm \text { SD of } \log _{\mathbf{1 0}} \text { CFU } \\
\text { in spleen of }\end{array}$} \\
\cline { 3 - 4 } & & Bs2WT & Vaccine \\
\hline Bs2 $\triangle w a d B$ & $10^{5}$ & $3.97 \pm 0.37^{\mathrm{a}, \mathrm{b}}$ & $2.61 \pm 0.97$ \\
Bs2 $\triangle p p d k$ & $10^{5}$ & $3.65 \pm 0.43^{\mathrm{a}, \mathrm{b}}$ & $2.39 \pm 0.54$ \\
Bs2 $\triangle p p d k \Delta w a d B$ & $10^{5}$ & $4.12 \pm 1.09^{\mathrm{a}, \mathrm{b}}$ & $2.68 \pm 1.17$ \\
Bs2 $\triangle w b k F$ & $10^{8}$ & $3.33 \pm 0.36^{\mathrm{a}, \mathrm{b}}$ & $2.45 \pm 0.56$ \\
Bs2 $\triangle w z m$ & $10^{8}$ & $2.83 \pm 1.88^{\mathrm{a}, \mathrm{b}}$ & $2.52 \pm 1.50$ \\
Rev1 & $10^{5}$ & $3.68 \pm 0.78^{\mathrm{a}}$ & $2.43 \pm 0.56$ \\
BSS & - & $6.69 \pm 0.13$ & \\
\hline
\end{tabular}

Statistical comparison $(n=5)$ of mean $\log _{10}$ Bs2WT CFU/spleen: ${ }^{a} p<0.001$ vs. BSS (unvaccinated) and ${ }^{\mathrm{b}} \mathrm{p}>0.05$ vs. Rev1. 
ruminant brucellosis in industrialized countries as well as to the fact that outbreaks in pigs can be controlled by hygienic measures and stamping out under the conditions prevailing in these countries [4]. Altogether, these circumstances have made less urgent the development of a $B$. suis vaccine. However, there is a reemergence of swine brucellosis in continental Europe (caused by $B$. suis bv 2) mainly because of the increase in production systems in which interaction with raising populations of wild boars becomes unavoidable and depopulation unfeasible. Although antibiotic treatment combined with culling may work for B. suis bv 2 outbreaks [47], this strategy cannot be generally applied and the use of antibiotics for these purposes is against WHO recommendations [48]. Moreover, it has been pointed out [4] that the huge change in pig breeding conditions taking place in many emerging economies and the fact that $B$. suis biovars 1 and 3 are highly zoonotic, create conditions where an effective $B$. suis vaccine would become very useful. Another reason for controlling the disease in pigs is that they can transmit the infection to cattle and that cows can excrete $B$. suis in milk multiplying the zoonotic risk [49].

Live attenuated rather than subcellular vaccines are of choice for developing new brucellosis vaccines [50], they are less costly to manufacture and, most important, mimic critical Brucella properties (cell invasion, trafficking, and suitable antigen presentation) needed to induce protective immunity. However, new brucellosis live vaccines should circumvent drawbacks posed by those currently used in ruminants (S19, Rev1 and RB51), particularly their potential to infect humans and resistance to antibiotics used in human brucellosis. We chose the LPS as a first target for developing an attenuated vaccine because this molecule is a key virulence factor of brucellae as well as an immunodominant antigen [51], and our current understanding of its genetics makes it feasible to obtain LPS mutants with a variety of phenotypes.

Concerning lipid A, the only Brucella lipid A mutant affected in the modified pathway (lptA) is not attenuated in mice [52], and no mutants in the conserved Raetz pathway [53] have been described in Brucella, possibly because they are not viable (Conde-Álvarez, Iriarte and Letesson, unpublished). On the other hand, the core oligosaccharide and the $\mathrm{O}$-chain genes are amenable to mutation. Previous investigations show that the structure of the LPS of at least B. abortus and B. melitensis is such that mutations in core glycosyltransferases can generate either $\mathrm{S}$ (i.e. O-chain bearing) or $\mathrm{R}$ (i.e. O-chain lacking) phenotypes (Figure 1B) $[18,29,30,54]$. In line with previous observations in $B$. melitensis and B. abortus, we found that $B$. suis $w a d B$ and $w a d D$ mutants carry a core oligosaccharide defect that generates attenuation while not disturbing the O-chain. Therefore, both mutants are a first type of LPS vaccine candidates, and as they display similar attenuation, we chose Bs $2 \Delta w a d B$ for the protection experiments. It has been repeatedly shown that $R$ mutants (i.e. lacking an O-chain linked to the core) of $\mathrm{S}$ brucellae are also attenuated and thus represent live vaccine candidates [19]. However, R mutants display various degrees of attenuation depending on the extent of core defect, and work with $B$. abortus suggests that maintaining an intact core is necessary for an optimal $R$ vaccine candidate [55]. As depicted in Figure 1A, B, there are two types of $\mathrm{R}$ mutants with a complete core: those devoid of O-chain (mutants in $w b o A, w b o B, w b k A, w b k E$ or $w b k F$ ) and those carrying cytoplasmic O-chain precursors (mutants in wadA and wzm/wzt) $[17,56]$. In this work, we tested a mutant of each kind (Bs2 $\Delta w b k F$ and Bs2 $\Delta w z m)$ and we found that they were cleared faster than $\mathrm{Bs} 2 \Delta w a d B$ or $\mathrm{Bs} 2 \Delta w a d D$ despite being inoculated at a dose 1000 fold higher, a result consistent with similar comparisons in B. abortus and B. melitensis. Attenuation was similar for Bs $2 \Delta w b k F$ and Bs $2 \Delta w z m$ but since the latter carries $\mathrm{O}$-chain precursors that have been reported to improve protection in an $\mathrm{R}$ background (see below), we tested both.

Despite their different LPS defects and degree of attenuation, we obtained similar protection with Bs $2 \Delta w a d B$, Bs $2 \Delta w b k F$, Bs $2 \Delta w z m$ and the Rev1 control. Although these results demonstrate protective immunity in mice, they have to be understood in the context of the experimental conditions. Indeed, because of the high costs and long-time span of experiments in natural hosts, mice have been used as a first step in the analyses of brucellosis vaccines (reviewed in [57]). However, it is important to keep in mind that the BALB/c mouse model commonly used for these purposes was originally developed for the biological control of master seeds of S19 and Rev1, and that results with vaccine candidates are affected by at least, dose of vaccine and challenge, challenge strain, and time span between vaccination and challenge, so that control by inclusion of S19 or Rev1 is critically important [57]. In this work, we have followed the recommended procedures for all these parameters [57], and the only bias in our comparisons is in the use of different doses, $10^{8} \mathrm{CFU}$ for the two $\mathrm{R}$ vaccines and $10^{5} \mathrm{CFU}$ for Bs $2 \Delta w a d B$ (or Bs $2 \Delta p p d K$, see below) and Rev1. These doses were chosen because of the consistent observation that $R$ vaccine candidates require 100 to 1000 fold higher doses than S19 or Rev1 to produce a comparable protection in mice $[17,58,59]$. However, controlled experiments in cattle or sheep with B. abortus or B. melitensis $\mathrm{R}$ mutants show that even with an increase in the dose they are not as effective as S19 or Rev1 [18, 19], indicating that B. abortus or B. melitensis R strains induce comparatively 
lower protection than $S$ vaccines. To know whether this limitation also applies to their B. suis homologues requires controlled experiments in swine. This is worth studying because an optimal $R$ vaccine should not interfere in diagnostic tests that use whole S Brucella cells (rose bengal and complement fixation) [60]. However, the presence of $\mathrm{O}$-chain precursors in Brucella $\mathrm{R}$ mutants has been shown to trigger antibodies interfering in all tests [60] and therefore, as the Bs $2 \Delta w z m$ did not yield better results, $\mathrm{Bs} 2 \Delta w b k F$ is a better candidate for a $B$. suis $R$ vaccine. Nevertheless, since all $R$ vaccines interfere in other tests (indirect and competitive ELISA, fluorescence polarization assay, etc.) [60] that are widely used, it is not immediately clear whether Bs $2 \Delta w b k F$ would be advantageous when compared with a $\mathrm{S}$ candidate like Bs $2 \Delta w a d B$ that might result in better protection in the natural host.

We also investigated a metabolic mutant. The rational for this strategy is to reduce multiplication by hampering $B$. suis central $C$ metabolism in pathways connecting PEP and TCA (tricarboxylic acid cycle) (Figure 1C). We have shown before that while both PckA and PpdK are involved in these steps in B. suis biovar 5, only PpdK is active in $B$. abortus [33]. Interestingly, the same mutation disabling $B$. abortus PckA is present in B. melitensis (see above and [33]), and we report here that B. suis bv2 $p c k A$ is also mutated albeit in a different position. Taken together, these observations strongly suggest that the function of PckA is irrelevant with regards to virulence in ruminants and swine. On the contrary, the single Bs $2 \Delta p p d K$ mutant was attenuated, and this is reminiscent of the attenuation observed for single $p p d K$ mutants of $B$. abortus in mice [34] and of B. melitensis ppdK in sheep (Zúñiga-Ripa, Conde-Álvarez, Iriarte, de Miguel, Arce-Gorvel, Gorvel, Moriyón, Blasco, and Muñoz, unpublished). Here, we also show that the attenuation generated by hampering $C$ metabolism at the PEP-TCA connection could be useful in the development of brucellosis vaccines. Since at a $10^{5} \mathrm{CFU}$ dose Bs $2 \Delta p p d K$ conferred the same level of protection as Rev1, it is also an interesting vaccine candidate. Although $S$ vaccines generate interference in serodiagnosis, the use of the conjunctival route has been shown to minimize the problem in ruminants $[20,60]$ and it could also be effective in pigs.

The literature shows that the reduction in mouse spleen colonization has been reported previously for several B. suis vaccine candidates. Nasal immunization with a recombinant adhesin (BtaF) in cyclic-di-AMP protected mice against $B$. suis bv1 administered intragastrically but not when challenged intratracheally [61]. However, the protocol of immunization and costs indicate that this vaccine when improved with BtaF synergic antigens [61] would be more suitable for human use. Strain VTRS1, an R wboA mutant of B. suis biovar 4 [22], when administered intravenously (IV), was more efficacious against B. suis bv4 than RB51 administered IP. However, the reference S19 vaccine (IV) yielded better results [22]. Later, a VTRS1 homologue (VTRS2) created in a B. suis biovar 1 (strain 1330) over-expressing the potentially immunocontraceptive multimeric $\mathrm{GnRH}$ protein was developed but this construct did not afford protection against $B$. suis 1330, even after a booster administration [23]. Also following the $\mathrm{R}$ vaccine approach, a $B$. suis biovar $1 \mathrm{R}$ mutant in pgm (Figure $1 \mathrm{~B}$ ) reduced spleen colonization in mice challenged with $B$. suis 1330 , but no reference vaccine control was included [21]. Also, pgm mutants carry an incomplete core and are defective in the synthesis of periplasmic $\beta$-glucans, a pleiotropic effect that may explain why comparison with $B$. melitensis showed that they were less protective than mutants with a full core [17].

RB51 has been manipulated in attempts to improve the poor performance observed in the above-summarized experiment. A first study [25] constructed derivatives over-expressing WboA (disrupted by IS711 in RB51) (Figure 1A, B illustrate the role WboA in LPS synthesis), the $\mathrm{L} 7 / \mathrm{L} 12$ ribosomal protein and the $\mathrm{Cu} / \mathrm{Zn}$ superoxide dismutase (SOD) in a leuB mutant of RB51 using plasmid pNS4 (which is stable because it complements the leuB defect) [62]. The best construct (RB51leuB/SOD/WboA) administered IP reduced B. suis 1330 spleen colonization but no reference vaccine control was included. In a more recent work [26], the expression of O-chain was further enhanced by over-expressing $w b o A$ and $w b k F$. The construct (RB51WboAKF) provided protection against $B$. suis 1330 similar to that of the wboA mutated $\mathrm{R} B$. suis VTRS1 [26]), and improved VTRS1 when administered at a higher dose. However, no reference vaccine was included in the experiments of protection against $B$. suis. The rational for restoring the O-chain in RB51 is not obvious because of the rifampicin resistance and infectivity for humans of RB51 [27] and also because it offsets the main potential advantage of $R$ mutants as vaccines (i.e. not triggering antibodies to the S-LPS).

Some vaccine candidates developed in a B. neotomae background have also been tested against $B$. suis in mice. Moustafa et al. [63] investigated gamma-inactivated $B$. neotomae, and two recombinants over-expressing Bp26 and SOD, administered twice IP conferred protection against $B$. suis 1330 . This study did not include a control with a standard vaccine seemingly because the main aim was to develop a human vaccine (in animals a booster immunization is most often difficult to implement). Similarly, because of the potential advantages of $\mathrm{R}$ vaccines with regards to some serological tests, a $B$. neotomae wboA mutant (strain $\mathrm{BNW}$ ) has been tested [24]. However, it is to be noted that, 
although $B$. neotomae is currently accepted as a BSL2 Brucella, there is increasing evidence of its zoonotic potential [64] and wide range [65].

The vaccine candidates described here afforded protection in mice similar to that obtained with Rev1 and, although the variety of experimental conditions make comparisons difficult, they seem to be in this regard similar or better than those in previous works. Indeed, optimal live brucellosis vaccines should show stable attenuation, lack antibiotic markers and be easy to discriminate from wild-type bacteria; moreover, they should not be infectious for humans. Since we generated attenuation by in-frame deletion, which makes the mutation irreversible and easy to detect by PCR, did not use antibiotic markers and circumvented the main issues related to infectiveness in humans by using $B$. suis bv2, these candidates seem to offer clear advantages. Indeed, live attenuated vaccines should have a time span of multiplication in the host long enough to elicit a protective immune response and be cleared soon enough to avoid abortions and excretion. Whether the multiplication and permanence in pigs of these attenuated candidates are adequate requires experiments in the natural host before they can be assessed for protection. In this regard, exploring the full potential of vaccines constructed in $B$. suis bv2 also requires investigation of their effectiveness against other $B$. suis biovars also infecting these domestic animals.

\section{Supplementary information}

Supplementary information accompanies this paper at https://doi. org/10.1186/s13567-020-00815-8.

\section{Additional file 1. Bacterial strains and plasmids.}

Additional file 2. B. suis 1330 and $B$. suis CITA 198 (Bs2WT) but not $B$. suis bv2 Thomsen are virulent in mice. Mice were inoculated intraperitoneally (IP) with $1 \times 10^{5} \mathrm{CFU} /$ mouse.

Additional file 3. Primers and PCR products expected in mutant construction.

Additional file 4. Oligonucleotide sequences used for Bs $2 \Delta w z m$ and Bs2 $\Delta w b k F$ mutant constructions.

Additional file 5. Differential characteristics of species of the genus Brucella and mutants.

Additional file 6. Bs2WT pckA frameshift is in a position different from that in other Brucella species that carry a mutated PckA. Alignment of PckA of B. microti and B. suis biovar 5, B. melitensis $16 \mathrm{M}$ and $B$. abortus 2308, B. suis bv2 Thomsen and Bs2WT. The amino acids differing are indicated in bold and shaded in gray.

Additional file 7. Splenomegaly induced by the Bs2 mutants investigated.

\section{Acknowledgements}

We are grateful to Christoph Dehio for the generous gift of the $\beta 2150$ cells and to Sara Serrano for excellent technical assistance.

\section{Authors' contributions}

RC-A, PM and IM conceived and coordinated the study. MI supervised the genomic studies. BA-A, MJdM, LL-A, MS-B and AZ-R, performed experiments. RC-A, BA-A, PM, and IM wrote the manuscript. All authors analyzed the results. All authors read and approved the final manuscript.

\section{Funding}

This research was supported by the Institute for Tropical Health funders (Obra Social la CAIXA -LCF/PR/PR13/11080005- and Fundación Caja Navarra, Fundación María Francisca de Roviralta, Ubesol and Inversiones Garcilaso de la Vega S.L) and grants from MINECO (AGL2014-58795-C4-1-R) and Aragón Government (Grupo de Investigación A13_17R). B.A.A. is the recipient of the Ph.D. Fellowship BES-2015-075609 funded by the MINECO.

\section{Ethics approval and consent to participate}

Procedures in mice were in accordance with the current European (directive 86/609/EEC) and Spanish (RD 53/2013) legislations, supervised by both Ethical Committee for Animal Experimentation of CITA and Animal Welfare Committee of the University of Navarra and authorized by Aragón (Reports No. 201420 and 2014-21) and Navarra (CEEA 045/12) Governments.

\section{Competing interests}

The authors declare that they have no competing interests.

\section{Author details}

${ }^{1}$ Instituto de Salud Tropical (ISTUN), Instituto de Investigación Sanitaria de Navarra (IdiSNA) and Dpto. de Microbiología y Parasitología, Universidad de Navarra, c/Irunlarrea 1, 31008 Pamplona, Spain. ${ }^{2}$ Unidad de Producción y Sanidad Animal, Centro de Investigación y Tecnología Agroalimentaria de Aragón (CITA), Avda. Montañana 930, 50059 Zaragoza, Spain. ${ }^{3}$ Instituto Agroalimentario de Aragón-IA2 (CITA-Universidad de Zaragoza), Zaragoza, Spain.

Received: 28 April 2020 Accepted: 5 June 2020

Published online: 23 July 2020

References

1. Whatmore AM (2009) Current understanding of the genetic diversity of Brucella, an expanding genus of zoonotic pathogens. Infect Genet Evol 9:1168-1184. https://doi.org/10.1016/j.meegid.2009.07.001

2. Alton GG, Jones LM, Angus RD, Verger JM (1988) Techniques for the brucellosis laboratory. Institut National de la Recherche Agronomique, Paris, p 190 (ISBN 2738000428)

3. Muñoz PM, MickV, Sacchini L et al (2019) Phylogeography and epidemiology of Brucella suis biovar 2 in wildlife and domestic swine. Vet Microbiol 233:68-77. https://doi.org/10.1016/j.vetmic.2019.04.025

4. EFSA (2009) Opinion of the panel of Animal Health and Welfare (AHAW) on a request from the commission on porcine brucellosis (Brucella suis). EFSA J. pp 1-12

5. Moreno E (2014) Retrospective and prospective perspectives on zoonotic brucellosis. Front Microbiol 5:213. https://doi.org/10.3389/fmicb .2014 .00213

6. Godfroid J, Scholz HC, Barbier T et al (2011) Brucellosis at the animal/ecosystem/human interface at the beginning of the 21st century. Prev Vet Med 102:118-131. https://doi.org/10.1016/j.prevetmed.2011.04.007

7. Crawford AB, Manthei CA (1948) Brucellosis of swine. U.S. Department of Agriculture, No. 781, pp 1-12

8. Kernkamp CH, Roepke MH (1948) Vaccination of pigs with Brucella abortus vaccine strain 19. J Am Vet Med Assoc 113:564-567

9. Lord VR, Cherwonogrodzky JW, Schurig GG et al (1998) Venezuelan field trials of vaccines against brucellosis in swine. Am J Vet Res 59:546-551

10. Stoffregen WC, Olsen SC, Bricker BJ (2006) Parenteral vaccination of domestic pigs with Brucella abortus strain RB51. Am J Vet Res 67:18021808. https://doi.org/10.2460/ajvr.67.10.1802

11. Cedro VCF, Casas-Olascoaga R, Garcia-Carrillo C et al (1977) Inmunidad que confieren las vacunas INTA, oleo-suis, oleo-Bang 2 y Brucella melitensis frente a B. suis. Rev Mil Vet 24:217-229

12. $\mathrm{WHO}$ (1999) The development of new/improved Brucellosis vaccines: report of WHO meeting. WHO/EMC/ZDI/98 14:1-48 
13. Peng C, Li YJ, Huang DS, Guan P (2020) Spatial-temporal distribution of human brucellosis in mainland China from 2004 to 2017 and an analysis of social and environmental factors. Environ Health Prev Med 25:1. https ://doi.org/10.1186/s12199-019-0839-z

14. Verger J-M, Grayon M, Zundel E et al (1995) Comparison of the efficacy of Brucella suis strain 2 and Brucella melitensis Rev. 1 live vaccines against a Brucella melitensis experimental infection in pregnant ewes. Vaccine 13:191-196. https://doi.org/10.1016/0264-410X(95)93135-V

15. Blasco JM, Marín C, Jiménez de Bagüés MP, Barberán M (1993) Efficacy of Brucella suis strain 2 vaccine against Brucella ovis in rams. Vaccine 11:1291-1294. https://doi.org/10.1016/0264-410X(93)90097-H

16. Edens JDFJ (1966) Immunization of swine with an endotoxin-containing preparation of Brucella suis. Am J Vet Res 27:1327-1331

17. González D, Grilló M-J, De Miguel M-J et al (2008) Brucellosis vaccines: assessment of Brucella melitensis lipopolysaccharide rough mutants defective in core and O-polysaccharide synthesis and export. PLoS One 3:e2760. https://doi.org/10.1371/journal.pone.0002760

18. Barrio MB, Grilló MJ, Muñoz PM et al (2009) Rough mutants defective in core and O-polysaccharide synthesis and export induce antibodies reacting in an indirect ELISA with smooth lipopolysaccharide and are less effective than Rev 1 vaccine against Brucella melitensis infection of sheep. Vaccine 27:1741-1749. https://doi.org/10.1016/j.vaccine.2009.01.025

19. Moriyón I, Grilló MJ, Monreal D et al (2004) Rough vaccines in animal brucellosis: structural and genetic basis and present status. Vet Res 35:1-38. https://doi.org/10.1051/vetres:2003037

20. Ducrotoy MJ, Muñoz PM, Conde-Álvarez R et al (2018) A systematic review of current immunological tests for the diagnosis of cattle brucellosis. Prev Vet Med 151:57-72

21. Czibener C, Del Giudice MG, Spera JM et al (2016) Delta-pgm, a new liveattenuated vaccine against Brucella suis. Vaccine 34:1524-1530. https:// doi.org/10.1016/j.vaccine.2016.02.025

22. Winter AJ, Schurig G, Boyle SM et al (1996) Protection of BALB/C mice against homologous and heterologous species of Brucella by rough strain vaccines derived from Brucella melitensis and Brucella suis biovar 4. Am J Vet Res 57:677-683

23. Smith GP, Jain-Gupta N, Alqublan H et al (2019) Development of an auxotrophic, live-attenuated Brucella suis vaccine strain capable of expressing multimeric GnRH. Vaccine. https://doi.org/10.1016/j.vaccine.2018.12.070

24. Jain-Gupta N, Waldrop SG, Tenpenny NM et al (2019) Rough Brucella neotomae provides protection against Brucella suis challenge in mice. Vet Microbiol 239:108447. https://doi.org/10.1016/j.vetmic.2019.108447

25. Rajasekaran P, Surendran N, Seleem MN et al (2011) Over-expression of homologous antigens in a leucine auxotroph of Brucella abortus strain RB51 protects mice against a virulent B. suis challenge. Vaccine 29:3106-3110. https://doi.org/10.1016/j.vaccine.2011.02.054

26. Dabral N, Burcham GN, Jain-Gupta N et al (2019) Overexpression of wbkF gene in Brucella abortus RB51WboA leads to increased O-polysaccharide expression and enhanced vaccine efficacy against B. abortus 2308, B. melitensis $16 \mathrm{M}$, and $B$. suis 1330 in a murine brucellosis $\mathrm{m}$. PLoS One 14:e0213587. https://doi.org/10.1371/journal.pone.0213587

27. Ashford DA, di Pietra J, Lingappa J et al (2004) Adverse events in humans associated with accidental exposure to the livestock brucellosis vaccine RB51. Vaccine 22:3435-3439

28. Iriarte M, González D, Delrue RM et al (2004) Brucella LPS: structure, biosynthesis and genetics. In: López-Goñi l, Moriyón I (eds) Brucella: molecular and cellular biology. Wymondham, Horizon Bioscience, pp 159-192

29. Salvador-Bescós M, Gil-Ramírez Y, Zúñiga-Ripa A et al (2018) WadD, a new Brucella lipopolysaccharide core glycosyltransferase identified by genomic search and phenotypic characterization. Front Microbiol 9:2293. https://doi.org/10.3389/fmicb.2018.02293

30. Gil-Ramírez Y, Conde-Álvarez R, Palacios-Chaves L et al (2014) The identification of $w a d B$, a new glycosyltransferase gene, confirms the branched structure and the role in virulence of the lipopolysaccharide core of Brucella abortus. Microb Pathog 73:53-59. https://doi.org/10.1016/j.micpa th.2014.06.002

31. Soler-Lloréns P, Gil-Ramírez Y, Zabalza-Baranguá A et al (2014) Mutants in the lipopolysaccharide of Brucella ovis are attenuated and protect against B. ovis infection in mice. Vet Res 45:72. https://doi.org/10.1186/s1356 7-014-0072-0
32. Barbier T, Zúñiga-Ripa A, Moussa S et al (2018) Brucella central carbon metabolism: an update. Crit Rev Microbiol 44:182-211. https://doi. org/10.1080/1040841X.2017.1332002

33. Zúñiga-Ripa A, Barbier T, Lázaro-Antón L et al (2018) The fast-growing Brucella suis biovar 5 depends on phosphoenolpyruvate carboxykinase and pyruvate phosphate dikinase but not on Fbp and GlpX fructose1,6-bisphosphatases or isocitrate lyase for full virulence in laboratory models. Front Microbiol 9:641. https://doi.org/10.3389/fmicb.2018.00641

34. Zúñiga-Ripa A, Barbier T, Conde-Álvarez R et al (2014) Brucella abortus depends on pyruvate phosphate dikinase and malic enzyme but not on Fbp and GlpX fructose-1,6-bisphosphatases for full virulence in laboratory Models. J Bacteriol 196:3045-3057. https://doi.org/10.1128/JB.01663 $-14$

35. Gerhardt P, Wilson JB (1948) The nutrition of Brucellae: growth in simple chemically defined media. J Bacteriol 56:17-24

36. Allard N, Garneau D, Poulin-Laprade D et al (2015) A diaminopimelic acid auxotrophic Escherichia coli donor provides improved counterselection following intergeneric conjugation with actinomycetes. Can J Microbiol 61:565-574. https://doi.org/10.1139/cjm-2015-0041

37. Figurski DH, Helinski DR (1979) Replication of an origin-containing derivative of plasmid RK2 dependent on a plasmid function provided in trans. Proc Natl Acad Sci USA 76:1648-1652. https://doi.org/10.1073/ pnas.76.4.1648

38. Scupham AJ, Triplett EW (1997) Isolation and characterization of the UDPglucose 4'-epimerase-encoding gene, galE, from Brucella abortus 2308. Gene 202:53-59

39. Martínez-Gómez E, Ståhle J, Gil-Ramírez Y et al (2018) Genomic insertion of a heterologous acetyltransferase generates a new lipopolysaccharide antigenic structure in Brucella abortus and Brucella melitensis. Front Microbiol 9:1092. https://doi.org/10.3389/fmicb.2018.01092

40. Choi K-H, Gaynor JB, White KG et al (2005) A Tn7-based broad-range bacterial cloning and expression system. Nat Methods 2:443-448. https:// doi.org/10.1038/nmeth765

41. Llobet E, March C, Gimenez P, Bengoechea JA (2009) Klebsiella pneumoniae OmpA confers resistance to antimicrobial peptides. Antimicrob Agents Chemother 53:298-302. https://doi.org/10.1128/AAC.00657-08

42. Garin Bastuji B, Bowden RA, Dubray G, Limet JN (1990) Sodium dodecyl sulfate-polyacrylamide gel electrophoresis and immunoblotting analysis of smooth-lipopolysaccharide heterogeneity among Brucella biovars related to A and M specificities. J Clin Microbiol. 28(10):2169-2174

43. Conde-Álvarez R, Arce-Gorvel V, Iriarte M et al (2012) The lipopolysaccharide core of Brucella abortus acts as a shield against innate immunity recognition. PLoS Pathog 8:e1002675. https://doi.org/10.1371/journ al.ppat.1002675

44. Tsai C-M, Frasch CE (1982) A sensitive silver stain for detecting lipopolysaccharides in polyacrylamide gels. Anal Biochem 119(1):115-119. https ://doi.org/10.1016/0003-2697(82)90673-x

45. Bowden RA, Cloeckaert A, Zygmunt MS et al (1995) Surface exposure of outer membrane protein and lipopolysaccharide epitopes in Brucella species studied by enzyme-linked immunosorbent assay and flow cytometry. Infect Immun 63:3945-3952. https://doi.org/10.1128/ |Al.63.10.3945-3952.1995

46. Moreno E, Moriyón I (2006) The genus Brucella. 5.3. The brucellaphages. Prokaryotes Vol 5 Proteobacteria. Springer-Verlag, Berlin

47. Dieste-Pérez L, Frankena K, Blasco JM et al (2016) Efficacy of antibiotic treatment and test-based culling strategies for eradicating brucellosis in commercial swine herds. Prev Vet Med 126:105-110. https://doi. org/10.1016/J.PREVETMED.2016.01.033

48. WHO (2014) Antimicrobial resistance: global antibiotic resistance surveillance report. World Health Organization, Geneva (ISBN 978924156474 8). http://apps.who.int/iris/bitstream/10665/112642/1/9789241564 748_eng.pdf

49. Ewalt DR, Payeur JB, Rhyan JC, Geer PL (1997) Brucella suis biovar 1 in naturally infected cattle: a bacteriological, serological, and histological study. J Vet Diagn Investig 9:417-420. https://doi.org/10.1177/10406 3879700900414

50. Pandey A, Cabello A, Akoolo L et al (2016) The case for live attenuated vaccines against the neglected zoonotic diseases brucellosis and bovine tuberculosis. PLoS Negl Trop Dis 10:e0004572. https://doi.org/10.1371/ journal.pntd.0004572 
51. Zhao Y, Arce-Gorvel V, Conde-Álvarez R, Moriyon I, Gorvel JP (2018) Vaccine development targeting lipopolysaccharide structure modification. Microbes Infect 20(9-10):455-460. https://doi.org/10.1016/j.micin f.2017.11.006

52. Conde-Álvarez R, Palacios-Chaves L, Gil-Ramírez Y et al (2018) Identification of IptA, IpxE, and IpxO, three genes involved in the remodeling of Brucella cell envelope. Front Microbiol 8:2657. https://doi.org/10.3389/ fmicb.2017.02657

53. Raetz $\mathrm{CRH}$, Reynolds CM, Trent MS, Bishop RE (2007) Lipid A modification systems in Gram-negative bacteria. Annu Rev Biochem 76:295-329. https ://doi.org/10.1146/annurev.biochem.76.010307.145803

54. Conde-Álvarez R, Arce-Gorvel V, Gil-Ramírez Y et al (2013) Lipopolysaccharide as a target for brucellosis vaccine design. Microb Pathog. https:// doi.org/10.1016/j.micpath.2012.11.011

55. Monreal D, Grilló MJ, González D et al (2003) Characterization of Brucella abortus O-polysaccharide and core lipopolysaccharide mutants and demonstration that a complete core is required for rough vaccines to be efficient against Brucella abortus and Brucella ovis in the mouse mo. Infect Immun 71:3261-3271. https://doi.org/10.1128/IAI.71.6.3261-3271.2003

56. Godfroid F, Cloeckaert A, Taminiau B et al (2000) Genetic organisation of the lipopolysaccharide O-antigen biosynthesis region of Brucella melitensis 16M (wbk). Res Microbiol 151:655-668. https://doi.org/10.1016/S0923 -2508(00)90130-X

57. Grilló M-J, Blasco J, Gorvel J et al (2012) What have we learned from brucellosis in the mouse model? Vet Res 43:29. https://doi. org/10.1186/1297-9716-43-29

58. Jiménez de Bagüés MP, Elzer PH, Jones SM et al (1994) Vaccination with Brucella abortus rough mutant RB51 protects BALB/C mice against virulent strains of Brucella abortus, Brucella melitensis, and Brucella ovis. Infect Immun 62:4990-4996

59. Stevens MG, Olsen SC, Pugh GW, Brees D (1995) Comparison of immune responses and resistance to brucellosis in mice vaccinated with Brucella abortus 19 or RB51. Infect Immun 63:264-270. https://doi.org/10.1128/ IAl.63.1.264-270.1995

60. Ducrotoy MJ, Conde-Álvarez R, Blasco JM, Moriyón I (2016) A review of the basis of the immunological diagnosis of ruminant brucellosis. Vet Immunol Immunopathol 171:81-102. https://doi.org/10.1016/j.vetim m.2016.02.002

61. Muñoz González F, Sycz G, Alonso Paiva IM et al (2019) The BtaF adhesin is necessary for full virulence during respiratory infection by Brucella suis and is a novel immunogen for nasal vaccination against Brucella infection. Front Immunol 10:1775. https://doi.org/10.3389/fimmu.2019.01775

62. Rajasekaran P, Seleem MN, Contreras A et al (2008) Brucella abortus strain RB51 leucine auxotroph as an environmentally safe vaccine for plasmid maintenance and antigen overexpression. Appl Environ Microbiol 74:7051-7055. https://doi.org/10.1128/AEM.01511-08

63. Moustafa D, Garg VK, Jain N et al (2011) Immunization of mice with gamma-irradiated Brucella neotomae and its recombinant strains induces protection against virulent $B$. abortus, B. melitensis, and B. suis challenge. Vaccine 29:784-794

64. Waldrop SG, Sriranganathan N (2019) Intracellular invasion and survival of Brucella neotomae, another possible zoonotic Brucella species. PLoS One 14:e0213601. https://doi.org/10.1371/journal.pone.0213601

65. Sidwell RW, Lundgren DL, Thorpe BD (1967) Experimental studies with four species of Brucella in selected wildlife, laboratory, and domestic animals. Am J Trop Med Hyg 16:665-674. https://doi.org/10.4269/ajtmh .1967 .16 .665

\section{Publisher's Note}

Springer Nature remains neutral with regard to jurisdictional claims in published maps and institutional affiliations.
Ready to submit your research? Choose BMC and benefit from:

- fast, convenient online submission

- thorough peer review by experienced researchers in your field

- rapid publication on acceptance

- support for research data, including large and complex data types

- gold Open Access which fosters wider collaboration and increased citations

- maximum visibility for your research: over 100M website views per year

At BMC, research is always in progress.

Learn more biomedcentral.com/submissions 